\title{
Performance of the improved larger acceptance spectrometer: VAMOS++
}

\author{
M. Rejmund ${ }^{\mathrm{a}}$ B. Lecornu ${ }^{\mathrm{a}}$ A. Navin ${ }^{\mathrm{a}}$ C. Schmitt ${ }^{\mathrm{a}}$ S. Damoy ${ }^{\mathrm{a}}$ O. Delaune ${ }^{\mathrm{a}}$ J.M. Enguerrand ${ }^{\mathrm{a}}$ \\ G. Fremont ${ }^{a}$ P. Gangnant ${ }^{\text {a }}$ L. Gaudefroy ${ }^{b}$ B. Jacquot ${ }^{\mathrm{a}}$ J. Pancin ${ }^{\mathrm{a}}$ S. Pullanhiotan ${ }^{\mathrm{c}}$ C. Spitaels ${ }^{\mathrm{a}}$ \\ ${ }^{\mathrm{a}}$ Grand Accélérateur National d'Ions Lourds (GANIL), CEA/DSM-CNRS/IN2P3, Bd Henri Becquerel, BP 55027, F-14076 Caen, France. \\ ${ }^{\mathrm{b}} C E A, D A M, D I F$, F-91297 Arpajon, France \\ ${ }^{\mathrm{c}}$ Inter University Accelerator Centre, Aruna Asaf Ali Marg, New Delhi, 110067, India
}

\begin{abstract}
Measurements and ion optic calculations showed that the large momentum acceptance of the VAMOS spectrometer at GANIL could be further increased from $\sim 11 \%$ to $\sim 30 \%$ by suitably enlarging the dimensions of the detectors used at the focal plane. Such a new detection system built for the focal plane of VAMOS is described. It consists of larger area detectors $(1000 \mathrm{~mm} \times 150 \mathrm{~mm})$ namely, a Multi-Wire Parallel Plate Avalanche Counter (MWPPAC), two drift chambers, a segmented ionization chamber and an array of Si detectors. Compared to the earlier existing system (VAMOS), we show that the new system (VAMOS++) has a dispersion-independent momentum acceptance. Additionally a start detector (MWPPAC) has been introduced near the target to further improve the mass resolution to $\sim 1 / 220$. The performance of the VAMOS++ spectrometer is demonstrated using measurements of residues formed in the collisions of ${ }^{129} \mathrm{Xe}$ at $967 \mathrm{MeV}$ on ${ }^{197} \mathrm{Au}$.
\end{abstract}

Key words: Magnetic spectrometer, Momentum acceptance, Particle identification

PACS: 29.30.-h, 25.70.-z

\section{Introduction}

Magnetic spectrometers allow the identification and characterization of products created in collisions of nuclei. In recent years, magnetic spectrometers in conjunction with Radioactive Ion Beams (RIB) produced from projectile fragmentation, have been used to explore nuclei far from stability [1]. The strong forward kinematic focusing of the fragments facilitates relatively modest acceptance spectrometers to collect and identify most of the fragments of interest. Unlike at energies $\geq 50 \mathrm{MeV} / \mathrm{u}$, relevant to projectile fragmentation, at energies around the Coulomb barrier, the collection and identification of the various reaction products is more challenging (due to the lower energies and larger angular distributions of the products). Thus the characterization of nuclei produced with low production cross sections requires a large acceptance spectrometer coupled with a suitable detection system. These are also necessary for exploiting re-accelerated beams from ISOL facilities. Among such modern large acceptance spectrometers along with suitable detectors, VAMOS [2] at GANIL, PRISMA [3] at LNL Legnaro and MAGNEX [4] at LNS Catania have facilitated significant advances in the domain of nuclear structure and reaction mechanism. The increased collection efficiency and unique particle identification have been fully exploited by coupling powerful $\gamma$-arrays like EXOGAM [5], CLARA [6] and the AGATA demonstrator [7] for spectroscopic studies of neutron rich nuclei to understand the effect of the isospin on the evolution of nuclear structure. In particular, deep inelastic/transfer reactions have been exploited at both LNL and GANIL for characterization of neutron rich nuclei $[8,9]$.

The VAMOS spectrometer at GANIL coupled with a versatile detection system, has a variable mode of operation whereby one can use it either as a standard dispersive spectrometer or as a zero degree recoil separator. Recently, the feasibility of a gas filled operation of VAMOS has also been demonstrated, for the detection of evaporation residues from fusion reactions [10]. As discussed in Ref. [2], when VAMOS is operated in a dispersive mode, the focal plane detection setup and an efficient ray-tracing technique can be used to reconstruct and identify on an event by event basis, the mass $M$, element $Z$ and charge state $q$, for the various nuclei produced in a reaction. This method has been applied successfully to select and identify the reaction products from deep inelastic/transfer reactions both in inverse kinematics using stable beams [11] and in direct kinematics with RIB from SPIRAL [12]. The VAMOS spectrome- 
ter has been coupled with a variety of ancillary detectors (and their combinations) like EXOGAM, TIARA, MUST2, DIAMANT and INDRA [13]. Such a powerful combination of detectors has allowed to fully exploit both stable and RIB beams at GANIL for studies ranging from single particle structure and collective properties of exotic nuclei to the equation of state of nuclear matter [13]. More recently, using VAMOS in conjunction with the EXOGAM $\gamma$-array, it was possible to uniquely identify the $M$ and $Z$ of a large number of fission fragments. This showed, for the first time, the possibility of studying the prompt $\gamma$ spectroscopy of individually identified light and heavy fission fragments [14]. The experimental success of using inverse kinematics with beams having $A \geq 200$ (due to larger kinetic energy of the fragments and kinematic focusing) and the unambiguous identification capability of VAMOS opens up new opportunities for exploring the prompt $\gamma$ spectroscopy of neutron rich nuclei produced by fission and also the investigation of the fission process itself [15].

Ion optic calculations showed that the acceptance of VAMOS was presently being limited by the size of the existing detectors at the focal plane and that it was possible to further improve the acceptance of the spectrometer by increasing their dimensions (mainly along the horizontal direction) [2]. Thus with the goal of reaching new limits of sensitivity and improved mass resolution of uniquely identified nuclei over a large range of masses, a new set of detectors of larger active area including an additional time of flight start detector (MWPPAC) has been constructed. These detectors have been characterized using the measurement of residues produced in the reaction of ${ }^{129} \mathrm{Xe}+{ }^{197} \mathrm{Au}$ at $967 \mathrm{MeV}$. In this manuscript we briefly highlight the salient features of the spectrometer VAMOS and discuss the motivation of upgrading it to its present version (VAMOS ++$)$. In the next section, the various components of the detection system are described. This is followed by the section describing the results on the identification of the various residues and those demonstrating the larger acceptance of the spectrometer VAMOS++.

\section{VAMOS spectrometer at GANIL}

VAMOS is a ray-tracing spectrometer where the complete identification of the particle is achieved from the measured quantities in the focal plane. The optical elements of the spectrometer consist of large aperture magnetic quadrupoles and a dipole for focusing and dispersing the charged particles. For such a large magnetic system, the ion beam optics becomes highly non-linear and a direct measurement of particle coordinates at the focal plane alone is insufficient to determine the momentum of the transmitted particles. This is determined by reconstructing the parameters of the trajectory at the target by ray-tracing on an event by event basis [2]. It is achieved by measuring the position and angular coordinates of the particle trajectory at the focal plane of the spectrometer and reconstructing

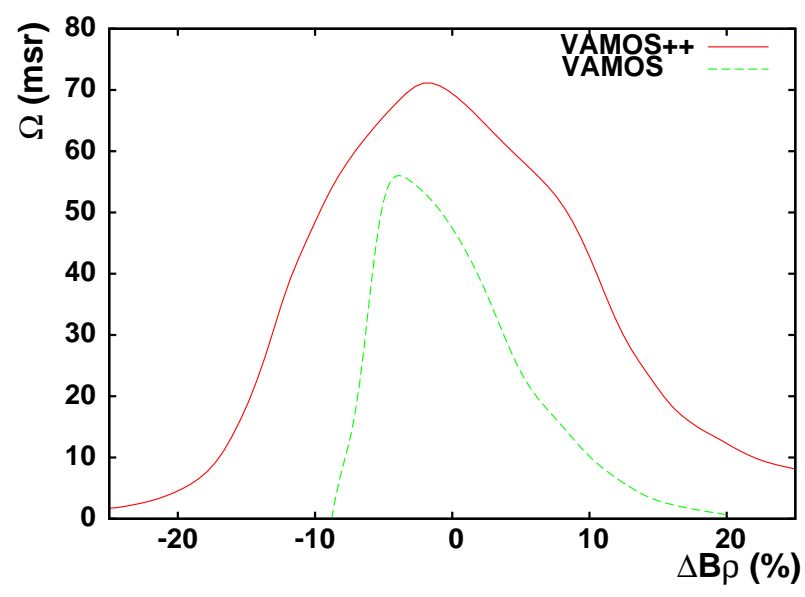

Figure 1. Calculated effective solid angle (msr) of VAMOS as a function of the relative rigidity $\Delta B \rho$, for two different sizes of the detection system, where $\Delta B \rho=\left(\left(B \rho / B \rho_{0}\right)-1\right) \times 100$ and $B \rho_{0}$ is the reference magnetic rigidity corresponding to particles moving along the axis of the spectrometer. The dashed curve corresponds to the existing size of the focal plane detection system of VAMOS $(400 \mathrm{~mm} \times 110 \mathrm{~mm})$. The full curve corresponds to the dimension of the newly constructed focal plane detection system VAMOS++ $(1000 \mathrm{~mm} \times 150 \mathrm{~mm})$.

the trajectory from an image plane back to its origin at the target. The transformation matrix depends on the optical properties of the spectrometer and also requires a precise knowledge of the transport of particles through the magnetic system. The reconstruction procedure applied in VAMOS uses a polynomial function to relate the parameters at the target with those at the image plane. Precise measurements of the position and angle at the image plane, energy loss $(\Delta E)$, residual energy $\left(E_{r}\right)$ and time of flight $(T O F)$ through the spectrometer are used for the complete identification of the reaction products [2]. The VAMOS focal plane consisted of a versatile combination of detectors, comprising of a pair of drift chambers for measurement of the position $(x, y)$, a fast timing detector (secondary electron based detector, SED) for TOF, a segmented ionization chamber for energy loss and a silicon wall detector for residual energy. Initially, all these detectors were constructed with an area of $400 \mathrm{~mm} \times 110 \mathrm{~mm}$.

\subsection{Limitation in the acceptance and need for a larger area detector system}

The VAMOS spectrometer is among those spectrometers having the largest acceptance in the world today. A detailed investigation of the acceptance of the spectrometer has been reported in our earlier work where it was shown that the transmission of the particles through VAMOS is non-uniform and varies within the accepted range of angles and momenta [2]. The study of the transmission was performed by tracing the particles through the magnetic fields using the ion optical code ZGOUBI [16] (the various physical constraints imposed by the apertures of the magnets and detector windows are incorporated into the 


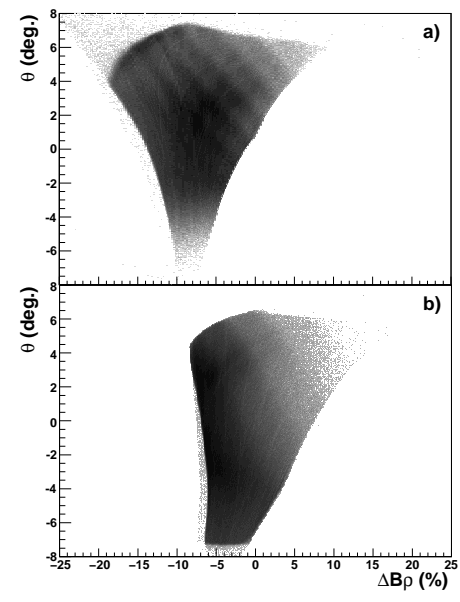

Figure 2. Two dimensional plot of $\theta$ as function of relative rigidity for transmitted particles for two positions of the existing detection system $(400 \mathrm{~mm} \times 110 \mathrm{~mm})$. a) The detectors were offset by $150 \mathrm{~mm}$ to lower $\mathrm{B} \rho$ with respect to the reference trajectory. b) The detectors were centred on the reference trajectory. The data were obtained using measurements for the ${ }^{208} \mathrm{~Pb}+{ }^{70} \mathrm{Zn}$ and ${ }^{238} \mathrm{U}+{ }^{48} \mathrm{Ca}$ systems respectively. The intensity pattern is governed by the reaction dynamics and is not of relevance here.

simulation). These simulations for studying the effect of the transmission as function of the size of the image plane were made for two different cases; one for an active area of $400 \mathrm{~mm} \times 110 \mathrm{~mm}$ (existing detector dimensions) and the other with a larger size of $1000 \mathrm{~mm} \times 150 \mathrm{~mm}$. The results for these two different sized image planes are compared in Fig. 1. The figure shows the effective solid angle (integrated over the accepted angular $(\theta, \phi)$ limits) plotted as a function of relative deviation of the magnetic rigidity $(\mathrm{B} \rho)$ of the transmitted particles with respect to the reference rigidity. The dashed (full) curve shows the solid angle distribution for a detector having a dimension of $400 \mathrm{~mm} \times 110 \mathrm{~mm}$ $(1000 \mathrm{~mm} \times 150 \mathrm{~mm})$. The figure clearly illustrates that with a larger detector dimensions there is a significant improvement in the transmission of the particles through the spectrometer. In the case of detectors with horizontal size of $1000 \mathrm{~mm}$, particles entering the spectrometer at different angles $(\theta, \phi)$ are now registered by the detectors over a larger range of $B \rho$, thereby extending the acceptance. (The dispersive direction of the spectrometer $(x)$ is indicated in Fig. 3 along with the coordinate system used for reconstruction of the particle trajectory.) The width of momentum acceptance at the corresponding half maximum could be used to quantify the difference between the two curves. Increasing the size of the detectors results in an increase of the momentum acceptance from $\sim 11 \%$ to $\sim 30 \%$. It should be noted that, in addition to the width of the above plot, the magnitude of the solid angle is also increased. The later is related to the increase in the vertical size of the detectors. An increase in the size of the detectors, results not only in particles within a larger momentum window being accepted, but also that the effective solid angle distribution becomes more symmetric and uniform. Calculations indicated that detectors of $1000 \mathrm{~mm} \times 150 \mathrm{~mm}$ size is suf-

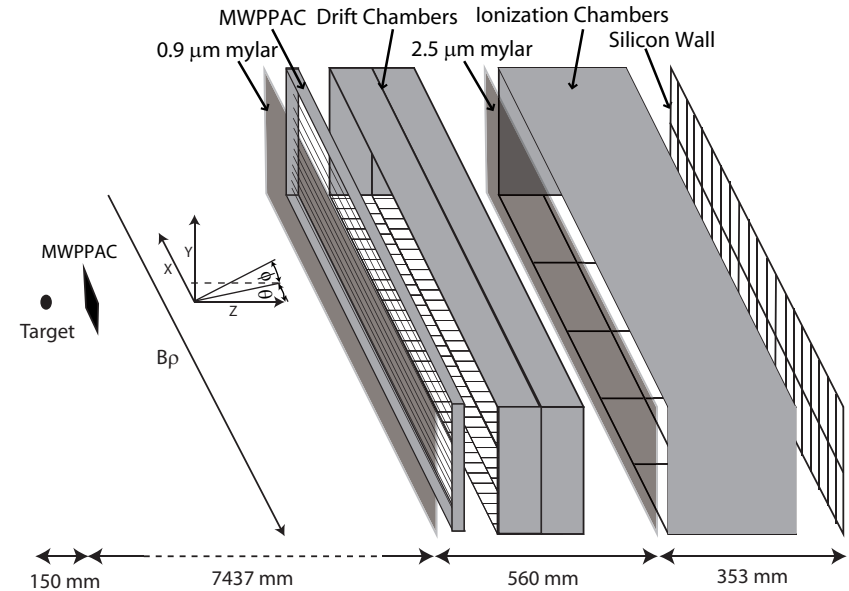

Figure 3. Schematic view of the new VAMOS focal plane detection system along with the MWPPAC near the target. The coordinate system used in the description of a trajectory is shown. The direction for increasing values of $\mathrm{B} \rho$ is also indicated.

ficient to record all transmitted trajectories satisfying the focal condition of the spectrometer.

The above observation was experimentally verified by performing a measurement using the existing smaller detectors $(400 \mathrm{~mm} \times 110 \mathrm{~mm})$, but displaced from the centre, mimicking larger sized detectors. In the experimental setup, all the focal plane detectors were shifted to the left of the reference trajectory. Products from deep inelastic/transfer reactions produced in the collision of a $1.29 \mathrm{GeV}$ beam of ${ }^{208} \mathrm{~Pb}$ on an enriched ${ }^{70} \mathrm{Zn}$ target were measured around the grazing angle. The range of rigidity and angle (in the reaction plane) of the particles registered by the detector is shown in Fig. 2a. Also shown below are the data for the ${ }^{238} \mathrm{U}+{ }^{48} \mathrm{Ca}$ system $[9,11]$ measured with the detectors centred on the reference trajectory. Figure 2a clearly demonstrates the increased transmission at lower relative rigidity. Simultaneously the $M$ and $M / q$ were successfully reconstructed. Based on these results a new large area detection system was designed and built. The new detectors along with changes made towards improving the time resolution of the system are described in the next section.

\section{New detection system for VAMOS}

The new detection system of VAMOS is composed of 4 types of detectors: $a$ ) Multi-Wire Parallel Plate Avalanche Counter (MWPPAC), b) Drift Chambers (DC), c) Segmented Ionization Chamber (IC) and $d$ ) 40 Silicon detectors $(\mathrm{Si})$ arranged in a wall like structure. The two DC measure the horizontal $(x)$ and vertical $(y)$ positions necessary to reconstruct the trajectories of the particles. The IC is used for the measurement of the energy loss $(\Delta E)$ and the $\mathrm{Si}$ for the residual energy $\left(\mathrm{E}_{r}\right)$. The time of flight is obtained using signals from the two MWPPAC, one placed close to the target and the other at the entry of the focal plane. This setup permits the measurement of all the parameters needed for the determination of the mass $M$, 

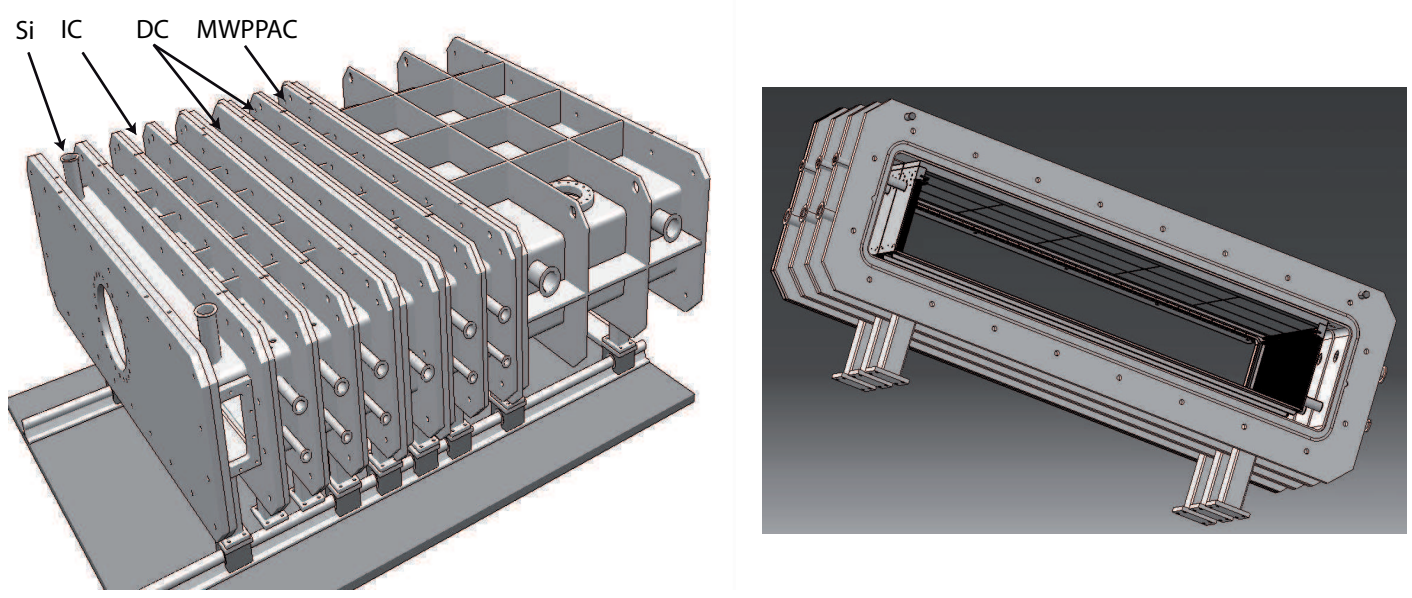

Figure 4. Modular mechanical set-up for the new detection system (left) and a single module containing the ionization chamber (right).

atomic number $Z$, charge state $q$, energy $E$ and angles at the target $\left(\theta_{l a b}, \phi_{l a b}\right)$ of the nuclei transmitted through the spectrometer. The detection system at the focal plane has an active area of $1000 \mathrm{~mm} \times 150 \mathrm{~mm}$. A schematic view of the system is shown in Fig. 3 . The chamber containing the focal plane detection system has also been changed. In the earlier configuration, the detectors and their preamplifiers were placed inside a single rectangular vacuum chamber. The detectors are now arranged in a modular manner as shown in Fig. 4. The new mechanical arrangement for the detectors allows for a precise and reproducible geometry. Each detector, attached to its own independent chamber, can be translated using rails. The mechanical structure allows for suitable placement of the detectors to minimize dead zones and thus reduce energy and angular straggling of the particles. In addition to making the mechanical operation easier, the relevant electronics are plugged on the PCB boards of the detectors in air (except for the Si detectors), minimizing the cable length and optimizing the heat dissipation. In the configuration used for the present work, the MWPPAC and the two DCs were operated with isobutane at a common gas pressure of 6 mbar. The segmented IC was operated between 20 to 40 mbar of $\mathrm{CF}_{4}$. A mylar foil $(2.5 \mu \mathrm{m})$ was used to separate the two gas volumes, while no foil was placed between the IC and the Si array.

\subsection{Multi-Wire Parallel Plate Avalanche Counter}

The choice of the MWPPAC, for fast timing, was based on the known good time resolution, high count rate capabilities and ease of operation of such a detector. Moreover the experience gained from operating such a device at PRISMA [17] helped in designing this large area detector. The MWPPAC constructed for the focal plane of VAMOS is composed of a central cathode and two anodes, each separated by $2.2 \mathrm{~mm}$. The cathode plane with vertical wires of $150 \mathrm{~mm}$ height and anode plane with $1000 \mathrm{~mm}$ long horizontal wires are made of gold coated tungsten wires. These wires have a diameter of $20 \mu \mathrm{m}$ and a pitch of $500 \mu \mathrm{m}$ and
$1 \mathrm{~mm}$ for the central and external plane respectively. The vertical wires (cathode) are polarized $(\sim-500 \mathrm{~V})$ whereas the horizontal wires (anode) are grounded. An entrance window $(0.9 \mu \mathrm{m}$ mylar) isolates the beam line vacuum. (If necessary another similar window can be used to isolate the other detectors.) The windows are supported by $100 \mu \mathrm{m}$ diameter nylon wires placed every $50 \mathrm{~mm}$. When an ion crosses the detector, the ionization electrons created in the two gaps are accelerated towards the anodes producing an electron avalanche and the corresponding ions are collected on the central plane. The $1000 \mathrm{~mm}$ cathode is segmented into 20 independent sections to reduce the capacitance and thus ensures a large amplitude and a fast rise time of the signal. These time signals are obtained from the segmented cathode through fast voltage amplifiers (ORTEC FTA820).

\subsection{The drift chambers}

Each drift chamber (DC) has an active volume of $1000 \times 150 \times 100 \mathrm{~mm}^{3}$. As described in Ref. [2], they consist of a drift gap of $150 \mathrm{~mm}$, where ionization occurs, and an amplification gap of $20 \mathrm{~mm}$ separated by a Frisch grid. The grid is made of gold coated tungsten wires of $50 \mu \mathrm{m}$ diameter with a pitch of $2.54 \mathrm{~mm}$. As shown in Fig. 5a, the amplification wires (gold coated tungsten), are at a distance of $5 \mathrm{~mm}$ from the cathode and $15 \mathrm{~mm}$ below the Frisch grid. The wires are $20 \mu \mathrm{m}$ in diameter with a pitch of $10 \mathrm{~mm}$. The cathode plane is made up of two rows, each consisting of 160 pads. There are 4 wires in front of each cathode row, as shown in Fig. 5b. The pads are made of gold plated strips $6.02 \mathrm{~mm}$ wide at a pitch of $6.4 \mathrm{~mm}$ along the dispersive direction. To improve the measurement of the position in between pads, the two cathode rows are offset by half a strip (see Fig. 5b). These pads are read by GASSIPLEX chips [18] directly plugged on the cathode and are outside the vacuum chamber. The stiffness of the $1000 \mathrm{~mm}$ long wires can be controlled from the edges of the detector thus avoiding the use of mechanical supports. Since the drift distance is large, 6 polarized horizontal 


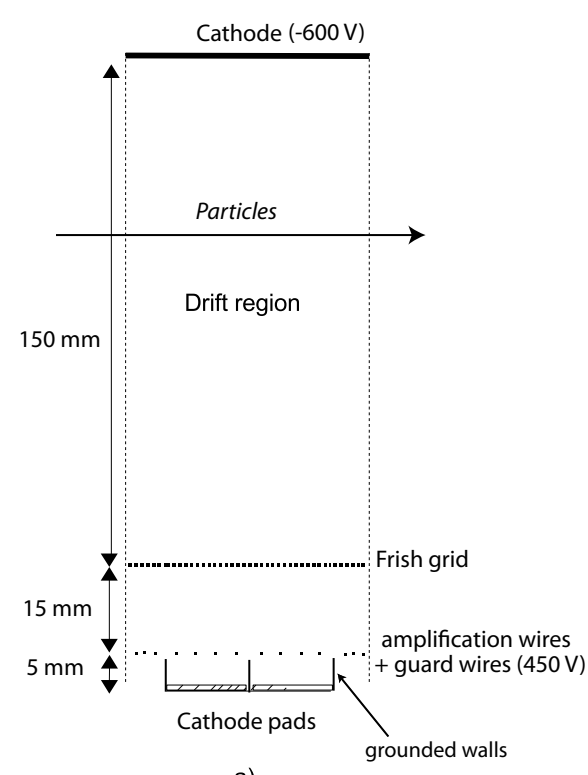

a)

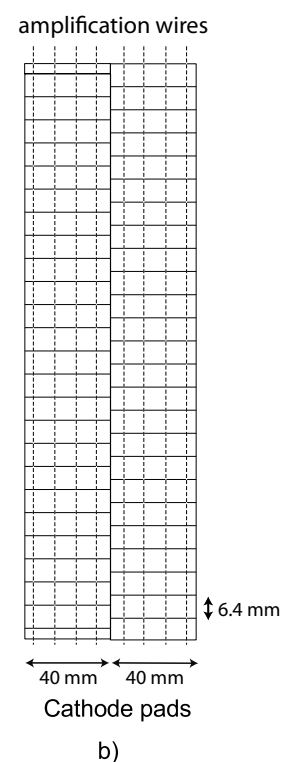

b)

Figure 5. Schematic view of a) the drift chamber in the Y-Z plane and $\mathrm{b}$ ) an expanded view of the the cathode pads in the $\mathrm{X}-\mathrm{Z}$ plane shown in a), the coordinate axes are shown in Fig. 3. Only a few of the total of 160 pads on two rows are shown for clarity.

metallic (guard) wires, placed at the entrance and exit of the DC ensure the homogeneity of the electric field in the drift region. The drift electrode is polarized negatively ( $\sim 100 \mathrm{~V} / \mathrm{mbar})$ while the Frisch grid is grounded. The amplification wires are positively polarized $(\sim 75 \mathrm{~V} / \mathrm{mbar})$ and the pads are grounded through the front-end cards. The design of the present drift chambers has been improved to some extent (as compared to that described in Ref. [2]). These improvements include $a$ ) the addition of wires in the dead zone at the entrance and exit of the DC (the area before and after the cathode pads in the Fig. 5a). These wires are polarized along with the amplification wires and allow the collection of all the ionization electrons created in this region. This reduces the uncertainty in the measurement of the drift time, $b$ ) grounded walls have been added in between the various cathode planes (in each drift chamber) to avoid crosstalk (Fig. 5a). Position resolutions of $\sim 270 \mu \mathrm{m}$ in the dispersive plane $(x)$ and $\sim 350 \mu \mathrm{m}$ in the drift direction $(y)$ were measured.

\subsection{The ionization chamber}

The ionization chamber (IC) is based on the standard design. It is made of three segments along the direction of the particle, each of which is further segmented into five pads as can be seen in Fig. 3. Each IC segment has an active volume of $1000 \times 120 \times 150 \mathrm{~mm}^{3}$ and is filled with $\mathrm{CF}_{4}$. The IC is composed of a drift gap of $150 \mathrm{~mm}$ between a drift cathode and a Frisch grid placed at $20 \mathrm{~mm}$ from the anode pads. The Frisch grid is made of gold coated tungsten wires of $50 \mu \mathrm{m}$ diameter with $1 \mathrm{~mm}$ pitch. An acceleration grid with the same characteristics is placed at a distance of $10 \mathrm{~mm}$ from the anode. At the entrance of the first segment of the

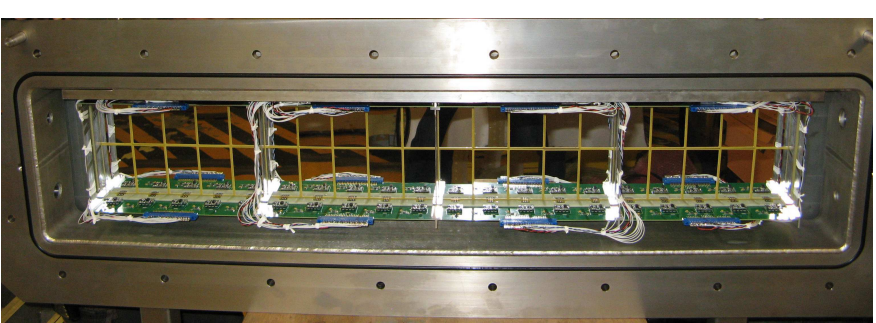

Figure 6. A picture of the $\mathrm{Si}$ wall (backside) showing the $40 \mathrm{Si}$ detectors, preamplifiers and detector housing.

IC, a grid of gold coated tungsten wires with $2.54 \mathrm{~mm}$ pitch is placed in between the drift cathode and the Frisch grid to ensure a good field homogeneity in the drift gap. This wire plane is duplicated at the exit of the third segment of the IC. The entrance window $(2.5 \mu \mathrm{m}$ mylar $)$ is supported by 32 vertical nylon wires to avoid any deformation arising due to different operating pressure for the DC and the IC. Typical pressures used varied between 20 and 40 mbar. A pad having a dimension of $1000 \mathrm{~mm} \times 20 \mathrm{~mm}$, placed at the entrance of the chamber, acts as a guard circuit to collect the ionization electrons created in first layer of the IC. The active pads of the first segment are thus smaller and have a dimension of $1000 \mathrm{~mm} \times 100 \mathrm{~mm}$. Typical polarization potentials of $22.5 \mathrm{~V} / \mathrm{mbar}$ in the drift gap and $9 \mathrm{~V} / \mathrm{mbar}$ between the grid and anode were used. Each pad is equipped with custom made charge preamplifiers having a sensitivity of $180 \mathrm{mV} / \mathrm{MeV}$ (Si equivalent).

\subsection{The silicon wall}

The silicon wall consists of a $20 \times 2$ matrix of 40 silicon detectors $(50 \mathrm{~mm} \times 80 \mathrm{~mm})$ with a $2 \mathrm{~mm}$ inactive space in between each of the detectors. The active area is thus $1000 \mathrm{~mm} \times 160 \mathrm{~mm}$. Each of the Si detectors is $500 \mu \mathrm{m}$ thick. The whole detector array is mounted along with the ionization chamber avoiding an exit window for the IC. Custom made charge preamplifiers with $2 \mathrm{mV} / \mathrm{MeV}$ sensitivity can be placed inside the vacuum chamber close to the detectors (higher gains of 4 or $20 \mathrm{mV} / \mathrm{MeV}$ are also available). Fig. 6 shows a dorsal view of the Si detectors, the preamplifiers along with the mechanical frame. The energy resolution for the detectors using an Am-Pu alpha source was found to be $\sim 60 \mathrm{keV}$ (FWHM). In the case when the thickness of the Si detectors is insufficient to stop the ions, other detectors can be mounted behind these detectors.

\subsection{The start detector}

To improve the time resolution for mass identification of the detected ions, a MWPPAC has been placed at the entrance of the spectrometer at a distance of $150 \mathrm{~mm}$ from the target. This detector rotates along with the spectrometer. It provides a signal for a time measurement, independent of the beam pulse, towards improving the mass resolution. The detector has an active area of $60 \mathrm{~mm} \times 60 \mathrm{~mm}$ 


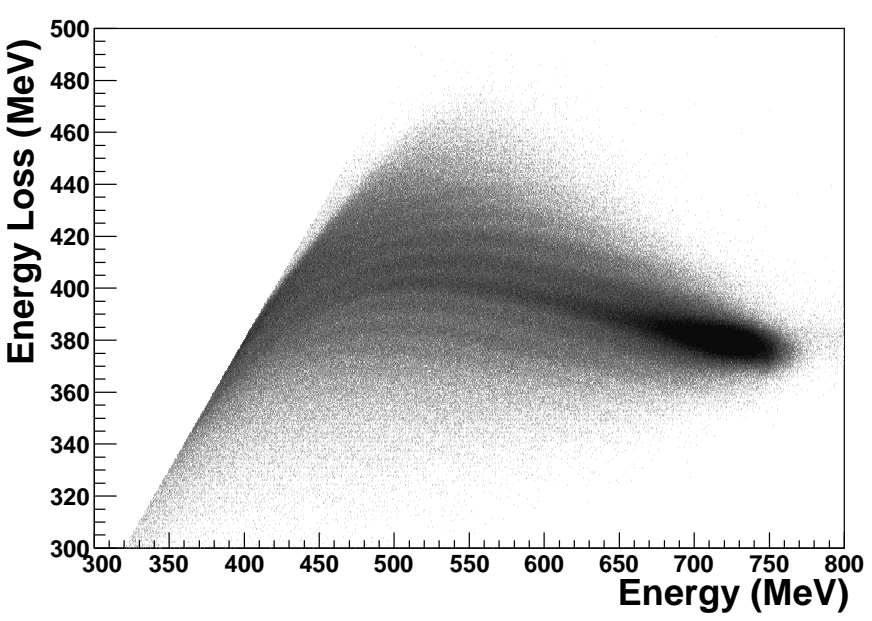

Figure 7. Two-dimensional spectrum of energy loss $(\Delta \mathrm{E})$ vs total energy $\left(\Delta \mathrm{E}+\mathrm{E}_{r}\right)$ measured over the full focal plane for the ${ }^{129} \mathrm{Xe}+{ }^{197} \mathrm{Au}$ system.

and is operated at a pressure of 6 mbar. The detector has two aluminized mylar windows $(0.9 \mu \mathrm{m}$ thick $)$. The active part of the detector is composed of 2 gaps of $2.4 \mathrm{~mm}$ width defined by three planes made up of gold coated tungsten wires having a $20 \mu \mathrm{m}$ diameter. Permanent magnets are placed outside the vacuum chamber to minimize the effect of the $\delta$ electrons created in the interaction of the beam in the target. The detector frame has a suitable size so as not to stop the direct beam when the VAMOS spectrometer is placed beyond $20^{\circ}$. This detector provides the start signal for each of the ion detected in the focal plane with a time resolution better than $500 \mathrm{ps}$ (FWHM). The time signal is read through a fast voltage amplifier from the central wire plane polarized at about $-500 \mathrm{~V}$ while the external planes are grounded.

\section{Performance of VAMOS ++}

The new detection system of VAMOS, described in the previous section, was commissioned by performing an experiment with a ${ }^{129} \mathrm{Xe}$ beam at $967 \mathrm{MeV}$ bombarding a 170 $\mu \mathrm{g} / \mathrm{cm}^{2}$ thick ${ }^{197} \mathrm{Au}$ target. The spectrometer was rotated to $40^{\circ}$ (around a calculated grazing angle) with respect to the beam direction. A typical beam intensity of $3 \mathrm{pnA}$ was used and the unreacted beam was stopped behind the quadrupole of VAMOS. The spectrometer was operated at a magnetic rigidity of $1.0352 \mathrm{~T}-\mathrm{m}$ in the momentum dispersive mode, with a dispersion of $2 \mathrm{~cm} / \%$, accepting reaction products emitted within the range $\theta_{l a b}=33^{\circ}-47^{\circ}$. Additionally 6 fully Compton suppressed EXOGAM $\gamma$-ray clover detectors were placed at a distance of $147 \mathrm{~mm}$ around the target at $90^{\circ}$ and $135^{\circ}$ relative to the beam axis, to measure the coincident $\gamma$-rays.

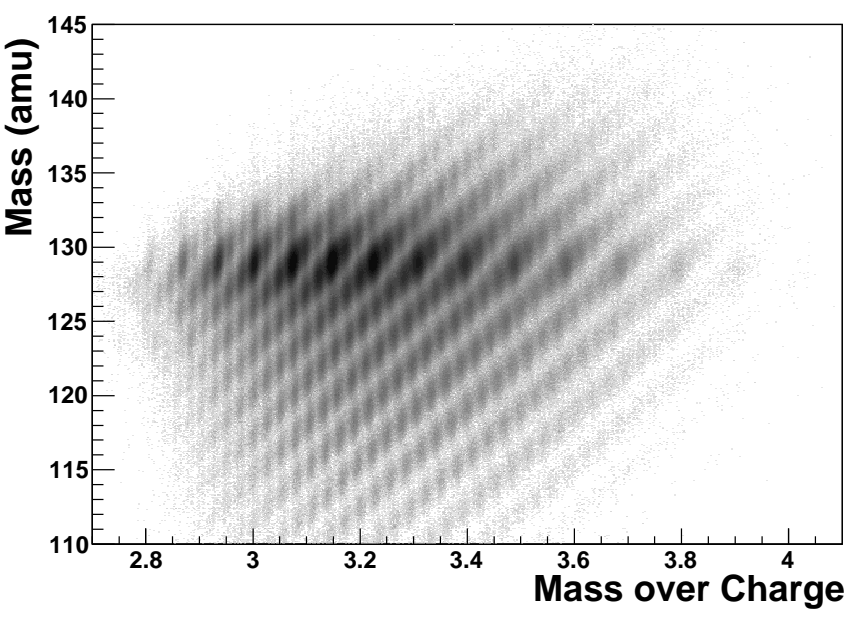

Figure 8. Spectrum showing the correlation between mass $(M)$ and mass over charge $(M / q)$ measured over the full focal plane for the ${ }^{129} \mathrm{Xe}+{ }^{197} \mathrm{Au}$ system.

\section{1. $M$ and $Z$ identification}

The ionization chamber was operated at a gas pressure of 30 mbar. This pressure was chosen to minimize the number of ions stopped in the ionization chamber. A typical $\mathrm{Z}$ identification plot displaying the energy loss $\Delta E$ in the ionization chamber as a function of total energy $\left(\Delta E+\mathrm{E}_{r}\right)$ is shown in Fig. 7. As can be seen from the figure, a variety of ejectiles produced in the reaction can be clearly identified. The most intense band corresponding to $\mathrm{Z}=54$ (beam) and the other reaction products arising from transfer and deep inelastic processes can be clearly resolved in the present setup. The resolution, $\Delta \mathrm{Z} / \mathrm{Z}$ was measured to be $\simeq 1 / 66$. The magnetic rigidity and the angle at the target of the reaction products were determined on an event by event basis by applying a reconstruction procedure, using a $10^{t h}$ order polynomial function $[2,19,20]$. The mass over charge $(M / q)$ and the mass $(M)$ were obtained independently using the reconstructed magnetic rigidity and a combination of the measured velocity and the total energy. A two dimensional spectrum of these parameters offers a unique and clean identification of the various masses accepted at the focal plane. Figure 8 shows such a two dimensional scatter plot. The individual masses having the same charge state form distinct (titled) bands correlating $M / q$ and $M$. These bands correspond to different charge states of the ions accepted by the spectrometer. It is seen that as many as 14 charge states (for $\mathrm{A}=129$ ) are accepted, which already demonstrates the uniqueness of this spectrometer. As can be seen from the figure, the masses belonging to different nuclei are well separated. Figure 9 shows a one dimensional mass spectrum displaying the large range of masses accepted in a single $\mathrm{B} \rho$ setting of VAMOS ++ . The mass resolution obtained here, demonstrates an improved measured resolution of $\Delta M / M$ of $\simeq 1 / 220$, as compared to our previously reported value of $\simeq 1 / 170$ [14]. This improved mass resolution was mainly achieved due to the bet- 


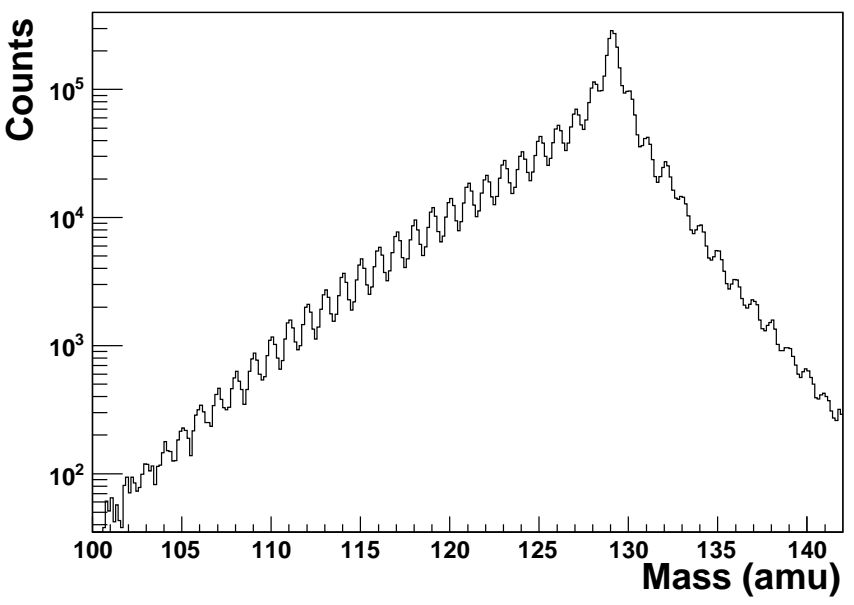

Figure 9. A typical mass spectrum of the fragments detected in the focal plane corresponding to Fig.8. The dominant peak corresponds to the mass of the projectile.

ter time resolution arising from the use of the MWPPAC start detector for obtaining the TOF. The rate handling limitation for the present setup arises due to the need of minimizing the pileup in the detectors and also the dead time of the data acquisition system. The count rate in the detector system is mainly restricted by the rate of the drift chambers (to be less than $10 \mathrm{kHz}$ ). The typical dead time of the acquisition system is $\sim 150 \mu$ s.

The new detection setup continues to be fully compatible with the measurement of coincidence $\gamma$-rays emitted by the moving reaction products. The coincident prompt $\gamma$ - rays corresponding to products identified at the focal plane were detected using EXOGAM clover detectors. The angle between the segment of the clover detector and the reconstructed velocity vector from the spectrometer was used to correct for the Doppler effect on an event by event basis. The use of $\gamma-\gamma$ coincidences for characterizing nuclei far from stability is severely restricted due to the low cross sections for such exotic channels. The excitation energy of the detected fragment can be constrained from its measured kinetic energy. Figure 10 shows such a $\theta_{\text {lab }}$ vs $E$ plot displaying the full kinematics of the reaction studied. The wide range of energies accepted by VAMOS++ in a single field setting can be seen. The two distinct regions visible in the figure corresponds to different reaction processes, quasi-elastic and those with a large energy damping. By selecting a suitable kinematic window parallel to the $Q=0$ line (Fig. 10), the excitation energy of the detected fragment can be constrained. It should be mentioned that only a relative excitation energy of the detected fragment can be obtained. Nevertheless this was successfully used to obtain the level scheme for exotic nuclei [9]. Additionally such a plot can also be transformed into a Wilczynsky plot to understand the energy dissipation in the reaction [21].

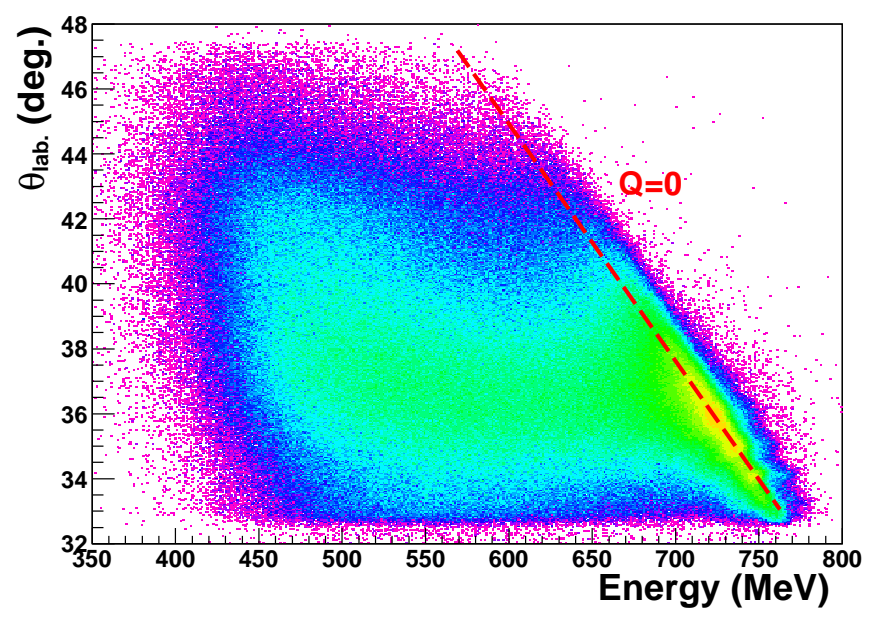

Figure 10. Spectrum showing the correlation between energy and $\theta_{\text {lab }}$ for the ${ }^{129} \mathrm{Xe}+{ }^{197} \mathrm{Au}$ system. The dashed line corresponds to the energy of the elastically scattered particles $(Q=0)$. This can be used to constrain the excitation energy of the detected fragment (see text).

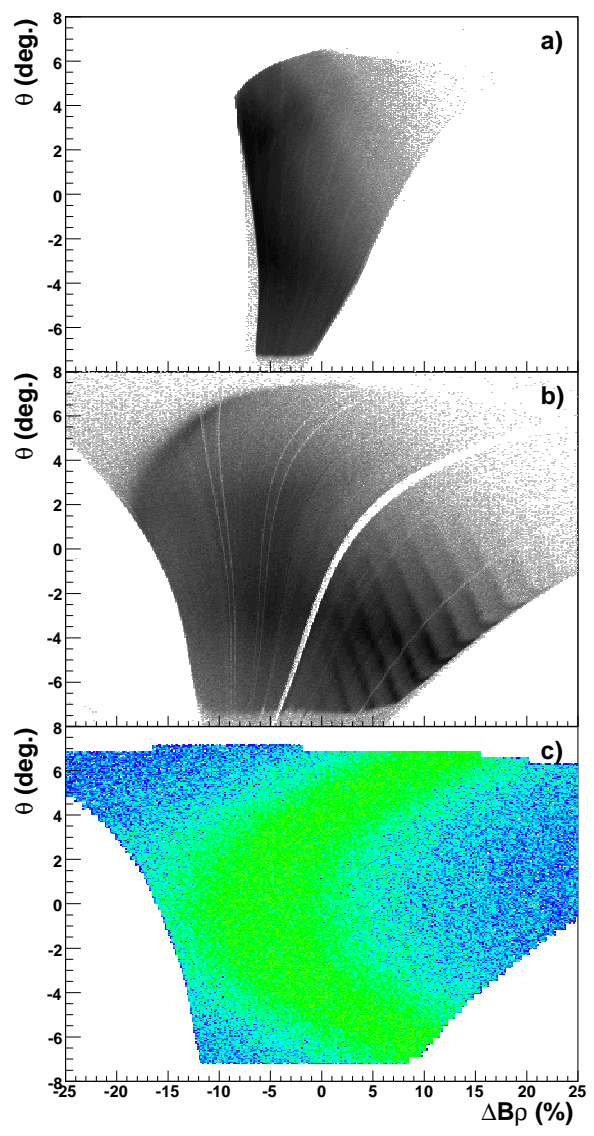

Figure 11. Two dimensional plot of $\theta$ as function of relative rigidity for transmitted trajectories. a) Same as in Fig 2b. for a detector size of $400 \mathrm{~mm} \times 110 \mathrm{~mm}$. b) From the present measurement with a detector size of $1000 \mathrm{~mm} \times 150 \mathrm{~mm}$. c) Simulation for an isotropic distribution of particles entering the spectrometer, for a detector size of $1000 \mathrm{~mm} \times 150 \mathrm{~mm}$. 


\subsection{Acceptance of VAMOS++}

new detection system was demonstrated in the previous section. Here we discuss the significant improvement in acceptance that has been achieved by increasing the size of the detectors. Fig. 11 shows a plot of the range of angle $\theta$ (in the reaction plane) plotted against the relative rigidity of the particles detected. The improved acceptance of VAMOS++ can be clearly seen. The upper most panel shows the range of the $(\theta-\mathrm{B} \rho)$ phase space accepted when the detector size was limited to $400 \mathrm{~mm} \times 110 \mathrm{~mm}$ (using the old detector setup [2]). Under such conditions, the $B \rho$ acceptance is limited to a range of around $-6 \%<\Delta B \rho<5 \%$ only, with a relative sharp fall at a lower rigidity (see also Fig. 1 and Fig. 2b). Figure 11b) shows the increase in the measured $(\theta-B \rho)$ phase space using the present large sized detection system. Compared to the use of the earlier smaller sized detectors, more particles entering the spectrometer with lower relative rigidities are now detected. The curved empty zone on the plot corresponds to the dead region arising due to a mechanical support in the centre of the MWPPAC chamber. (This support structure has been subsequently modified.) Figure 11c) shows for a comparison the same as that in b) but for a simulation of an isotropic distribution of particles entering the spectrometer. (Figure 13 of Ref. [2] presents a similiar plot for the previous detection system, clearly showing the incomplete and asymmetric nature of the acceptance.) A good agreement between the overall contours for the measurement and the simulation can be seen. As compared to the isotropic distribution used in generating the events shown in Fig. 11c), those in Fig. 11a) and b) results from a non isotropic distribution due to the reaction mechanism. Figure 11c) shows that the region of maximum transmission (having a parabolic shape) is fully within the acceptance and symmetric in $\theta$. The acceptance of VAMOS + + is now independent of the deviation angle of the dipole (dispersion independent) and is restricted only by the physical dimensions of the quadrupoles and those of the vacuum chambers of the subsequent optical elements.

\section{Conclusions}

We have presented the performance of the new detection system of the large acceptance VAMOS++ spectrometer at GANIL. With this increased $B \rho$ acceptance and better mass resolution, VAMOS ++ is expected to be the most sensitive spectrometer to select and identify exotic nuclei produced with low cross sections in peripheral and more central collisions. This large efficiency and selectivity, combined with other auxiliary detectors can be used to characterize these products both for understanding the evolution of nuclear structure and the reaction mechanism. The VAMOS ++ spectrometer is also expected to be a key device for tagging reaction products with the next generation ISOL facility SPIRAL2. The relevant changes required to utilize the high intensity beams of fission fragments are be- ing studied.

\section{Acknowledgements}

The authors would like to thank the GANIL management for their full support and encouragement for this project. They also acknowledge the strong support of the GANIL technical staff towards the smooth running of the various facilities and would like to thank F. Daudin for the mechanical design. We would also like to thank Y. Watanabe, S. Jeong and N. Imai for help in data taking. One of us (P. S) acknowledges financial support through the LIA France-India agreement.

\section{References}

[1] H. Geissel, G. Munzenberg and H. Weick, Nucl Phys A202 (2002) 701.

[2] S. Pullanhiotan, M. Rejmund, A. Navin, W. Mittig, S. Bhattacharyya, Nucl. Instr. and Meth. A593 (2008) 343.

[3] A.M. Stefanini et al., Nucl. Phys. A701 (2002) 217c; D. Montanari et al., Eur. Phys. J. A47 (2011) 4.

[4] A. Cunsolo et al., Nucl. Instr. and Meth. A637 (2002) 48; A. Cunsolo et al., Intl. Symposium on the Physics of Unstable Nuclei (ISPUN07), Hoi An, Vietnam, 3-7 July 2007; World Scientific Publishing Co. (2008) p. 26; M. Cavallaro et al., Nucl. Instr. and Meth. A637 (2011) 77.

[5] J. Simpson et al., Acta Physica Hungarica New Series: Heavy Ion Physics 11, (2000) 159.

[6] A. Gadea et al., Eur. Phys. J. A20, (2004) 193.

[7] E. Farnea et al., Nucl. Instr. and Meth. A621 (2010) 331.

[8] N. Marginean et al., Phys. Lett. B633 (2006) 696.

[9] M. Rejmund et al., Phys. Rev. C76 (2007) 021304(R).

[10] C. Schmitt et al., Nucl. Instr. and Meth. A621 (2010) 558.

[11] S. Bhattacharyya et al., Phys. Rev. Lett. 101 (2008) 032501.

[12] G. Benzoni et al., Eur. Phys. J. A45 (2010) 287.

[13] A. Navin, F. de Oliveira Santos, P. Roussel-Chomaz and O. Sorlin, J. Phys. G: Nucl. Part. Phys. G 38 (2011) 024004.

[14] A. Shrivastava et al., Phys. Rev. C80 (2009) 0515305(R).

[15] M. Caamano et al., Proc. $4^{\text {th }}$ Int. Workshop Nuclear Fission and Fission-Product Spectroscopy, Cadarache, France, 13-16 October 2009, A. Chatillon et al., Eds.; AIP Conf. Proc. 1175 (2010) p.15.

[16] G.F. Meot, Nucl. Instr. and Meth. A427 (1999) 353.

[17] S. Beghini et al., Nucl. Instr. and Meth. A 551 (2005) 364.

[18] J.C Santiard et al., CERN-ECP/ 94-17 (1994).

[19] S. Pullanhiotan, A. Chatterjee, B. Jacquot, A. Navin, M. Rejmund, Nucl. Instr. and Meth. B 266 (2008) 414.

[20] L. Gaudefroy and M. Rejmund (in preparation).

[21] J. Wilczyiski, Phys. Lett. B47 (1973) 484. 